\section{N1-Prostatakarzinom: Androgenentzug plus RT?}

Patienten mit pathologisch befallenen Lymphknoten nach radikaler Prostatektomie erhalten in der Regel eine alleinige Androgenentzugstherapie. In kleineren Serien war jedoch eine zusätzliche Strahlentherapie (RT) von Vorteil. Auch die Ergebnisse einer großen aktuellen Datenanalyse sprechen dafür.

R eim Prostatakarzinom lassen sich Dach radikaler Prostatektomie in den regionalen Lymphknoten bei bis zu $30 \%$ der Fälle Metastasen nachweisen. Bisher galt, dass befallene Lymphknoten auf eine generalisierte Erkrankung hinweisen und deswegen eine adjuvante Strahlentherapie nicht sinnvoll sei. Bei Patienten mit nodal negativen Hochrisikotumoren erwies sich die adjuvante Strahlentherapie jedoch als vorteilhaft. Auch in kleinen Serien mit allerdings ausgedehnter Lymphonodektomie schien sie das Überleben zu verbessern. Es blieb aber unklar, ob man dieses Ergebnis verallgemeinern kann. Nun wurden retros- pektiv Daten von 2.569 Patienten aus der National Cancer Database ausgewertet, die von 2003 bis 2011 radikal prostatektomiert worden waren und nodale, aber keine Fernmetastasen aufwiesen (N1M0). Zusätzlich zur Andogenentzugstherapie (ADT) erhielten 906 von ihnen (35,3\%) eine adjuvante RT. Nach median 53 Monaten betrug die 5-Jahres-Überlebensrate $87 \%$ bei den bestrahlten versus $82 \%$ bei den nur mit ADT behandelten Patienten $(p=0,007)$. Auch die multivariate Analyse bestätigte den lebensverlängernden Effekt der Strahlentherapie (Hazard Ratio [HR] 1,5; p < 0,001). Mit einem schlechteren Überleben assoziiert waren insbesondere Patienten mit $\geq 3$ befallenen Lymphknoten, positiven Resektionsrändern, Tumoren $\geq \mathrm{pT} 3$ und einem Gleason-Score $>7$ (p jeweils $<0,05)$.

In einer Matched-Pair-Analyse von 826 Patienten war die 5-Jahres-Überlebensrate bei den bestrahlten Männern erneut signifikant höher ( 88 vs. $81 \%$, HR $1,43 ; \mathrm{p}=0,004)$.

Fazit: Nach radikaler Prostatektomie und bei Befall regionaler Lymphknoten führt die zusätzliche RT im Vergleich zur alleinigen ADT zu einer signifikanten Überlebensverlängerung. Auch wenn diese Analyse retrospektiv war, kann sie angesichts der großen Zahl der Patienten helfen, für den individuellen Patienten die richtige postoperative Therapie zu finden.

Brigitte Schalhorn

Jegadeesh $\mathrm{N}$, et al. The role of adjuvant radiotherapy in pathologically lymph node positive prostate cancer. Cancer. 2017; 123(3): 512-20.

\title{
Prostatakarzinom: posttherapeutischer 6-Monats-PSA-Wert als Prädiktor
}

\begin{abstract}
Die Kombination aus Radiotherapie und Androgenentzugstherapie hat die Prognose des Prostatakarzinoms mit intermediärem oder hohem Risiko deutlich verbessert. Dennoch kommt es bei vielen Patienten im weiteren Verlauf zu Fernmetastasen. Ein zuverlässiger Marker könnte von Vorteil sein, um früh eine intensivierte Therapie zu initiieren.
\end{abstract}

n dieser Studie sollte geprüft werden, ob sich das prostataspezifische Antigen (PSA) sechs Monate nach Radiotherapie (RT) plus Androgenentzugstherapie (ADT) als früher Prädiktor für das Über- leben eignet. Retrospektiv wurden 532 Patienten mit intermediärem oder hohem Risiko analysiert, die von 1996-2012 Hochdosis-RT mit 78 Gy in 39 oder 70 Gy in 28 Fraktionen plus ADT erhielten.

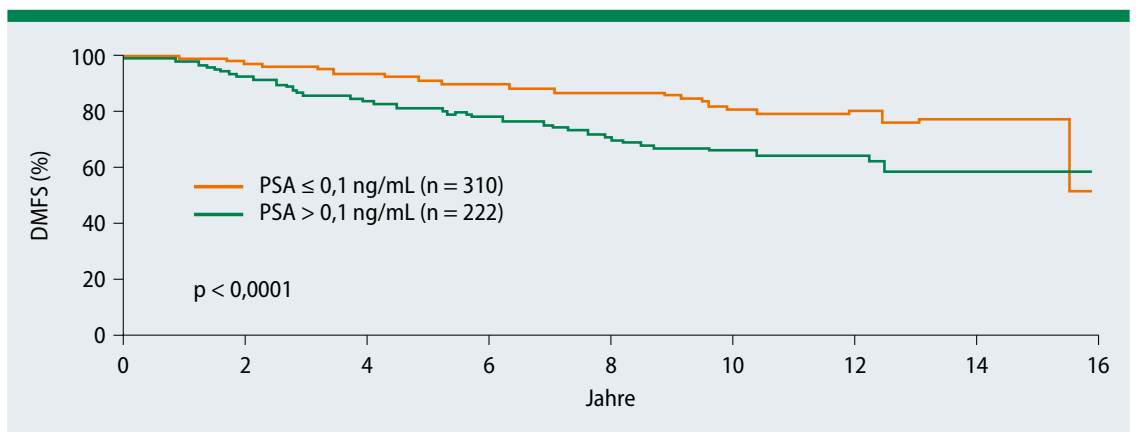

Abb.: Ein höherer PSA-Wert sechs Monate nach Therapie ging mit einem deutlich schlechteren fernmetastasenfreien Überleben (DMFS) einher.
Der mediane initiale PSA-Wert (iPSA) betrug $13 \mathrm{ng} / \mathrm{mL}$. Die ADT wurde bei $24,8 \%$ adjuvant durchgeführt, bei $72,9 \%$ neoadjuvant oder gleichzeitig. Der mediane PSA-Wert lag sechs Monate nach $\mathrm{RT}$ bei $0,1 \mathrm{ng} / \mathrm{mL}$. In der multivariaten Analyse war ein Wert über $0,1 \mathrm{ng} / \mathrm{mL}$ ein unabhängiger Prädiktor für ein kürzeres biochemisches rezidivfreies Überleben (Hazard Ratio [HR] 2,5; p < 0,0001), fernmetastasenfreies Überleben (HR 3,7; $\mathrm{p}<$ 0,0001; Abb.) und Prostatakarzinom-spezifisches Überleben (HR 5,4; $\mathrm{p}<0,0001)$.

Fazit: Der PSA-Wert nach sechs Monaten kann nach definitiver RT und gleichzeitiger ADT helfen, Patienten mit höherem Progressionsrisiko zu erkennen, um sie frühzeitig einer Salvage-Therapie zuzuführen.

Brigitte Schalhorn

Naik M, et al. Posttreatment prostate-specific antigen 6 months after radiation with androgen deprivation therapy predicts for distant metastasis-free survival and prostate cancer-specific mortality. Int J Radiation Oncol Biol Phys. 2016; 96(3): 617-23. 